\title{
FACE DETECTION AND RECOGNITION USING BACK Propagation Neural Network ANd Fourier GABOR FILTERS
}

\author{
Anissa Bouzalmat ${ }^{1}$, Naouar Belghini ${ }^{2}$, Arsalane Zarghili ${ }^{3}$ and Jamal Kharroubi ${ }^{4}$ \\ ${ }^{1}$ Department of Computer Sciences, Sidi Mohamed Ben Abdellah University, Fez, \\ Morocco \\ anissabouzalmat@yahoo.fr \\ ${ }^{2}$ Department of Computer Sciences, Sidi Mohamed Ben Abdellah University, Fez, \\ Morocco \\ n.belghini@ieee.ma \\ ${ }^{3}$ Department of Computer Sciences, Sidi Mohamed Ben Abdellah University, Fez, \\ Morocco \\ zarghili@yahoo.fr \\ ${ }^{4}$ Department of Computer Sciences, Sidi Mohamed Ben Abdellah University, Fez, \\ Morocco \\ jamal.kharroubi@yahoo.fr
}

\begin{abstract}
Face recognition is a field of computer vision that uses faces to identify or verify a person. In this paper, we present a neural network system for face recognition. Feature vector based on Fourier Gabor filters are used as input of the Back Propagation Neural Network (BPNN). To extract the features vector of the whole face in image, we use an algorithm for detecting skin human faces in color images and then we introduce Gabor filters with 8 different orientations and 5 different resolutions to get maximum information. Experiments show that the proposed method yields results.
\end{abstract}

\section{KEYWORDS}

Face Detection, Face Recognition, Bilinear Interpolation, Fourier Transform, Gabor Filter, Neural Network

\section{INTRODUCTION}

Human face detection and recognition has attracted much attention, it is an active area of research spanning several disciplines such as computer vision and automatic access control system. Especially, face detection is an important part of face recognition as the first step of automatic face recognition.

A complete face recognition system presents two stages. First detect the human faces in a cluttered background and extract pertinent features, second involve classification of facial images obtained in the previous stage. A robust face recognition system is a system based on good feature extraction method and good classifier. Neural network have been successfully applied to many pattern classification problems. And among techniques used in the literature for skin detection based on different color spaces include HSV, normalized RGB, YCrCb, YIQ and CIELAB. According to Sandeep et al. [1], HSV gives higher accuracy for skin pixel detection. DOI : $10.5121 /$ sipij.2011.2302 
Signal \& Image Processing : An International Journal (SIPIJ) Vol.2, No.3, September 2011

For feature extraction, it is proven that Gabor filters can extract the maximum information from local image regions [2][3] and it is invariant against, translation, rotation, variations due to illumination and scale [4][5][6].

In this study, we present a neural network system for face recognition. We use HSV space for skin detection, Gabor filters and Fourier transform for feature selection. Theses feature vectors are used as input of our Back Propagation Neural Network (BPNN), it was chosen as classifier for the proposed system because of its simplicity and its capability in supervised pattern recognition [7].

This paper is organized as follows: Description of our proposed solution is presented in section 2, in section 3, we gives the experimental results and conclusion.

\section{THE PROPOSED SOLUTION}

\subsection{The system architecture}

The system proposed in this research is designed for facial face recognition. The system consists of three modules: a) Facial feature extraction using Gabor filter b) dimensionality reduction using average value. Finally, the obtained feature vectors are fed up into BPNN for classification. (The overall system architecture is shown in Fig 1)



Figure 1. Description of the proposed solution architecture.

\subsection{Method of detection}

Face detection provides interesting challenges to the underlying pattern classification and learning techniques and many techniques [8], [9] have reported for locating skin color regions in the input image so, We are interested in skin detection .

this process is typically used as a preprocessing step to find regions that potentially have human faces and involves color histogram for skin (in the HSV space), the details of implementation are described in [1]. First, we detect the skin of the original image, then we reduce the image result in size $32 \times 32$ by bilinear interpolation. This is necessary to reduce the computation time.

\subsection{Features vector computation}

Gabor is a function that satisfies certain mathematical requirements extraction information which based on the use of a bank of Gabor filters [10], 8 orientations and 5 resolutions. The 2D Gabor filter function is the product of a $2 \mathrm{D}$ Gaussian and a complex exponential function. That satisfies 
certain mathematical requirements extraction information is formed by modulating a complex sinusoid by a Gaussian function where each filter is defined by:

$$
\text { Gabor }_{2 D}(x, y, \mu, v)=\frac{\|k \mu v\|^{2}}{\sigma^{2}} \exp \left(\frac{-\left\|k_{\mu v}\right\|^{2}\left(x^{2}+y^{2}\right)}{2 \sigma^{2}}\right)\left(\exp (i k \mu v *(x, y))-\exp \left(\frac{-\sigma^{2}}{2}\right)\right)
$$

Where:

$(\mathrm{x}, \mathrm{y})$ : The 2-dimensional input point.

$\mu$ : The orientation of the Gabor kernel

$v$ : The scale of the Gabor kernel.

II.II: The norm operator.

$\sigma:$ The standard deviation of the Gaussian window in the kernel.

$\mathrm{K} \mu \nu$ is The wave vector defined as:

$\mathrm{k}_{\mu \nu}=\frac{k \max }{f^{v}} \exp \frac{\pi \mu}{8}$

if 8 different orientations are chosen. Kmax is the maximum frequency, and $\mathrm{f} v$ is the spatial frequency between kernels in the frequency domain. In our configuration, 5 different scales and 8 orientations of Gabor wavelets are used, e.g. $v €\{0, \ldots, 4\}$ and $\mu €\{0, \ldots, 7\}$. Gabor wavelets are chosen with the parameters:

$$
k_{\max }=\frac{\pi}{2}, f=\sqrt{2}, \quad \sigma=\pi
$$

The collection of all 40 Gabor kernels is called a filter bank. An example can be found in Fig 2 .

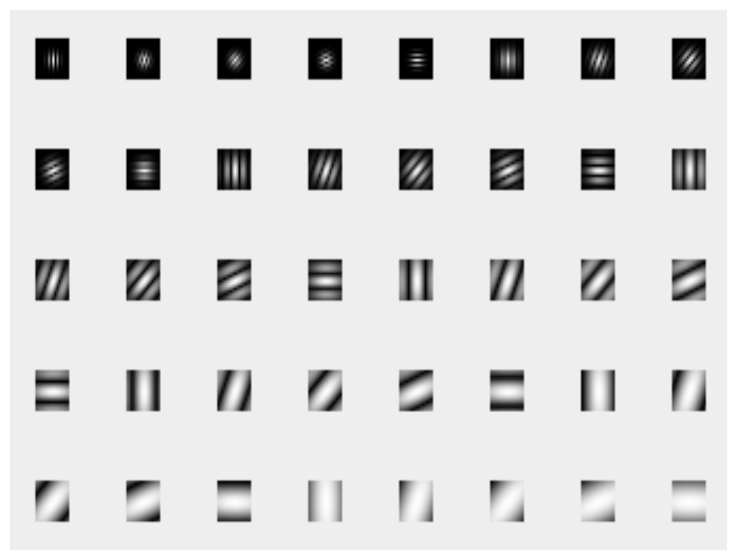

Figure 2. Gabor filters of size $16 \times 16$ by 8 orientations and 5 Resolutions (real part).

The Fourier Gabor wavelet representation of an image is the convolution of the Fourier image with the Fourier filter bank. The convolution of Fourier image F(I) and a Fourier Gabor kernel $\mathrm{F}\left(\operatorname{Gabor}_{\mu, v}(\mathrm{x}, \mathrm{y})\right)$ is defined as follows: 
Signal \& Image Processing : An International Journal (SIPIJ) Vol.2, No.3, September 2011

$$
\text { Convolution }_{\mu \nu}(x, y)=F(I(x, y)) * F\left(\operatorname{Gabor}_{\mu \nu}(x, y)\right)
$$

and called Fourier Gabor feature. As the response Convolution $\mu, v(\mathrm{x}, \mathrm{y})$ to each Fourier Gabor kernel is a complex function with a real part : Real $\{$ Convolution $\mu, v(\mathrm{x}, \mathrm{y})\}$ and an imaginary part : $\operatorname{Imag}\{$ Convolution $\mu, v(\mathrm{x}, \mathrm{y})\}$, we use its real $\operatorname{Real}\{$ Convolution $\mu, v(\mathrm{x}, \mathrm{y})\}$ to represent the Fourier Gabor features. The complete set of Gabor wavelet representations of the image I(x,y) is:

$$
O(I)=\{\text { Convolution } \mu \nu(x, y): \mu \in\{0, . ., 7\}, v \in\{0, . ., 4\}\}
$$

The resulting features for each orientation, scale are referred to as Fourier Gabor feature vector. The respectful steps in the algorithm is explained using the representation of the face with Fourier-Gabor filters

- Algorithm 1:

Step 1. Prepare $5 \times 8$ matrix Gabor each of size $16 \times 16$ as shown (Fig 2).

Step 2. Apply the Fourier transform to each matrix Gabor.

Step 3. Apply Fourier to each image in the training set of size $32 \times 32$.

Step 4. Convolution of the Fourier transform of the image size $32 \times 32$ by each image of the Fourier transformed Gabor size $16 \times 16$ ( 8 orientations and 5 scales) .

Step 5. Construct the image Fourier_Gabor_IMG $(5 \times 8 \times 32 \times 32)$ from the sub images $(32 \times 32$ obtained in step 4) (Fig 3).

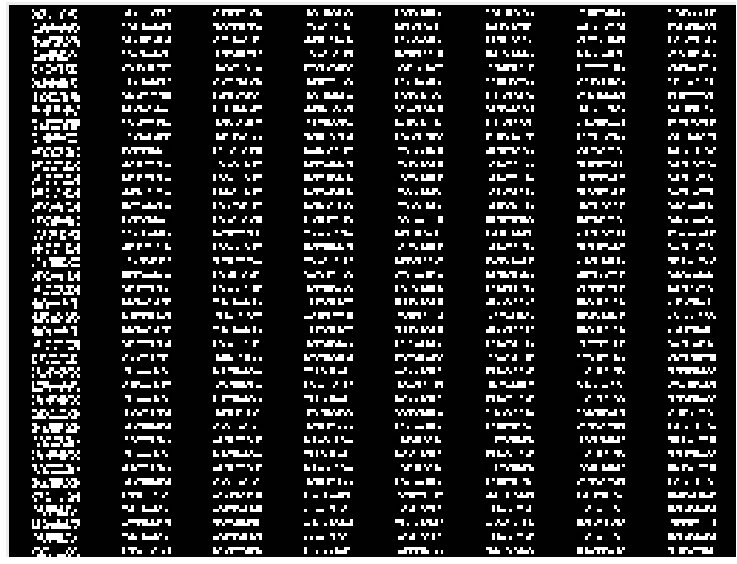

Figure 3: Fourier_Gabor_IMG $(5 \times 8 \times 32 \times 32)$ Results Convolution of Fourier transformed image $(32 \times 32)$ for the Fourier transformed of each Gabor filter $16 \times 16$.

The use of Gabor filters is very expensive in computing time, due to the convolution of the whole image with filter size $16 \times 16$. For this reason, we limit the use of the image size of $32 \times 32$ convolved with 40 Gabor filters: 8 orientations and 5 scales, then we resize the image result (Fourier_Gabor_IMG) to Result_IMG(100*100) of one dimension, and finally we reduce the dimension of extracted vector using average values:

We choose an incremental rate p, so the number of sample (numS) becomes 10000/p then we calculate the result vector for this channel: 
Signal \& Image Processing : An International Journal (SIPIJ) Vol.2, No.3, September 2011

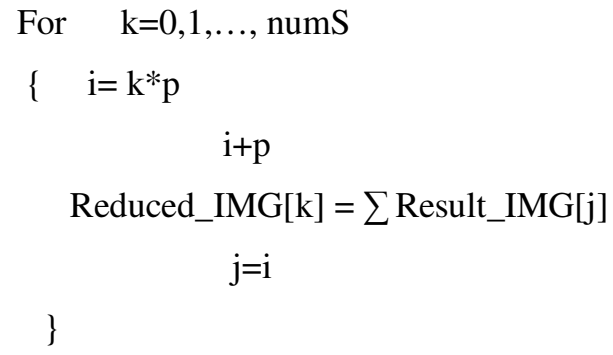

\subsection{The BPNN learning process}

After computation of the reduced vectors of dataset, back propagation algorithm is used to train our neural network. The process learning is as follow:

- Read data and specify the desired output for each vector.

- Randomly initialize weights and bias.

Then gradually adjust the weights of the network by performing the following procedure for all vectors:

- Compute the hidden layer and output layer neuron using sigmoid activation function.

- Calculate errors of the output and hidden layers.

- Adjust weights:

$$
w_{j i}(n)=w_{j i}(n-1)+\eta \delta_{j}(n) y_{i}(n)+\alpha \Delta j i(n-1)
$$

With:

$$
\delta_{j}(n)=\left\{\begin{array}{c}
e_{j}(n) y_{j}(n)\left[1-y_{j}(n)\right] \quad \text { If } j \in \text { output layer } \\
y_{j}(n)\left[1-y_{j}(n)\right] \sum_{k} \delta_{k}(n) w_{k j}(n) \quad \text { If } j \in \text { hidden layer }
\end{array}\right.
$$

$\eta$ and $\alpha$ are learning rate and momentum factor.

- Calculate to total error of network.

We repeat these steps on all patterns until minimizing the squared mean error.

\section{EXPERIMENTAL RESULTS}

In experimental results, we test with different images having simple backgrounds, different sizes and skin colours, on a sample XM2VTSDB [11]. The results (Fig 4) are good since we have a little change in different expression variations and pose. 
Signal \& Image Processing : An International Journal (SIPIJ) Vol.2, No.3, September 2011

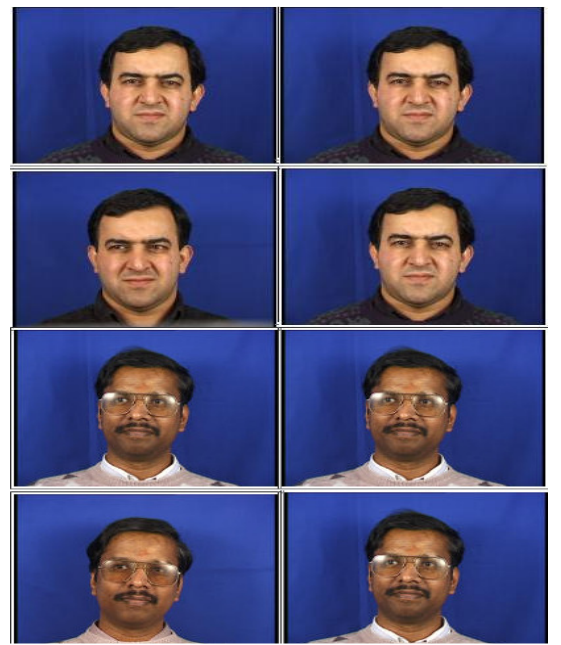

Figure 4: Example of input (left) and output (right) of our system

\section{CONCLUSION}

We have used a specific method for generating features vector of the whole face in an image, by first detecting face regions using the color of skin which presents a robust overlooked in different background, accessory and clothing. It is a fast algorithm for extracting human faces in color images and easy to implement. And second introducing Gabor filters to extract the maximum of information by varying the resolution and orientation. BPNN is then applied to perform the recognition task. This solution was implemented using Java environment. Results indicate that the proposed method achieves good results.

\section{REFERENCES}

[1] K. Sandeep, A.N. Rajagopalan,"Human Face Detection in Cluttered Color Images Using Skin Color and Edge Information”, ICVGIP Proceeding, 2002.

[2] H. Deng, L. Jin, L. Zhen, and J. Huang. A new facial expression recognition method based on local gabor filter bank and pca plus lda. International Journal of Information Technology, 11(11):86-96, 2005 .

[3] L. Shen and L. Bai. Information theory for gabor feature selection for face recognition. Hindawi Publishing Corporation, EURASIP Journal on Applied Signal Processing, Article ID 30274, 2006.

[4] J Essam Al Daoud, "Enhancement of the Face Recognition Using a Modified Fourier-Gabor Filter”,Int. J. Advance. Soft Comput. Appl., Vol. 1, No. 2, 2009.

[5] Z. Y. Mei, Z. Ming, and G. YuCong. Face recognition based on low diamensional gabor feature using direct fractional-step lda. In Proceedings of the Computer Graphics, Image and Vision: New Treds (CGIV'05), IEEE Computer Society,2005.

[6] B. Schiele, J. Crowley, ”Recognition without correspondence using mul-tidimensional receptive field histograms",International Journal on Com-puter Vision.36:3152,2000.

[7] Christopher M Bishop, "Neural Networks for Pattern Recognition” London, U.K.:Oxford University Press, 1995. 
Signal \& Image Processing : An International Journal (SIPIJ) Vol.2, No.3, September 2011

[8] H. Martin Hunke, Locating and tracking of human faces with neural network, Master's thesis,University of Karlsruhe, 1994.

[9] Henry A. Rowley, Shumeet Baluja, and Takeo Kanade. "Neural network based face detection,"IEEE Transactions on Pattern Analysis and Machine Intelligence, 20(I), pp.23-38, 1998.

[10] B. Schiele and J. Crowley. "Recognition without correspondence using multidimensional receptive field histograms". International Journal on Computer Vision, 36:3152, 2000.

[11] K Messer, J Matas, J Kittler, J Luettin, and G maitre, ” Xm2vtsdb: The extended m2vts database”, In Second International Conference of Audio and Video-based Biometric Person Authentication, March 1999. 